\title{
Posterior Segment Manifestations of Pathological Myopia: A Review
}

\author{
Ayman G Elnahry ${ }^{1 *}$ and Joseph H Talbet $^{2}$ \\ ${ }^{1}$ Department of Ophthalmology, Cairo University, Egypt \\ ${ }^{2}$ Howard University, USA
}

Submission: December 12, 2018; Published: January 04, 2019

*Corresponding author: Ayman Elnahry, Department of Ophthalmology, Faculty of Medicine, Cairo University, Cairo, Egypt

\begin{abstract}
Pathological myopia is a major growing public health concern in many countries worldwide including Egypt. Its exact cause is unknown, but multiple genetic and environmental factors are thought to play a role in its pathogenesis. The main concern with pathological myopia from a vitreoretinal perspective is the development of various posterior segment pathologies including tessellation, chorioretinal degeneration, lacquer cracks, posterior staphyloma, myopic choroidal neovascularization, macular holes, myopic foveoschisis, retinal detachment, and peripheral retinal pathologies such as lattice degeneration and snail track degeneration. In this article, we review the different posterior segment manifestations associated with pathological myopia and their morphological and clinical characteristics.
\end{abstract}

Keywords: Chorioretinal atrophy; Fuchs' spot; Lattice degeneration; Myopic CNV; Myopic retinopathy; Pathological myopia; Peripheral retinal lesions; Posterior segment manifestations; Posterior staphyloma; Tessellated fundus

Abbreviations: RPE: Retinal Pigment Epithelium; CNV: Choroidal Neovascularization; OCT: Optical Coherence Tomography; RRD: Rhegmatogenous Retinal Detachment; PVD: Posterior Vitreous Detachment; FTMH: Full Thickness Macular Hole; OLMH: Outer Lamellar Macular Hole; ILMH: Inner Lamellar Macular Hole; MRS: Macular Retinoschisis; ILM: Internal Limiting Membrane; MH: Macular Hole.

\section{Introduction}

Pathological myopia is a major cause of blindness worldwide that is becoming increasingly more prevalent in many countries of the world including Egypt [1,2]. Its exact cause is unknown; however, multiple genetic and environmental factors are thought to play a role in its complex pathogenesis [3]. The hallmark of pathological myopia is excessive and progressive axial length elongation which leads to degenerative changes in the sclera, choroid, and retinal pigment epithelium and compromises visual function $[4,5]$. Clinically, these changes include tessellated fundus, lacquer cracks, atrophy of the retinal pigment epithelium and choroid, choroidal neovascular membrane, posterior staphyloma, Fuchs' spot, macular hole, foveoschisis, retinal breaks and detachments, and peripheral retinal lesions [6]. The effect of these manifestations on the visual performance of patients with pathological myopia can be substantial which emphasizes the value of the correct diagnosis and management of these complications. In this article, we review the clinical and morphological characteristics of the various posterior segment manifestations that can occur in pathological myopia as well as the value of multimodal imaging in the diagnosis and management of these manifestations.

\section{Posterior segment manifestations of pathological my- opia}

\section{Myopic retinopathy}

Myopic retinopathy refers to a cluster of signs that indicate degeneration of chorioretinal tissues associated with the excessive axial elongation of the myopic eye. According to the Blue Mountains Eye Study, myopic retinopathy is defined as the presence of staphyloma, lacquer cracks, Fuchs' spot and chorioretinal thinning or atrophy [7].

\section{Tessellated (Tigroid) fundus}

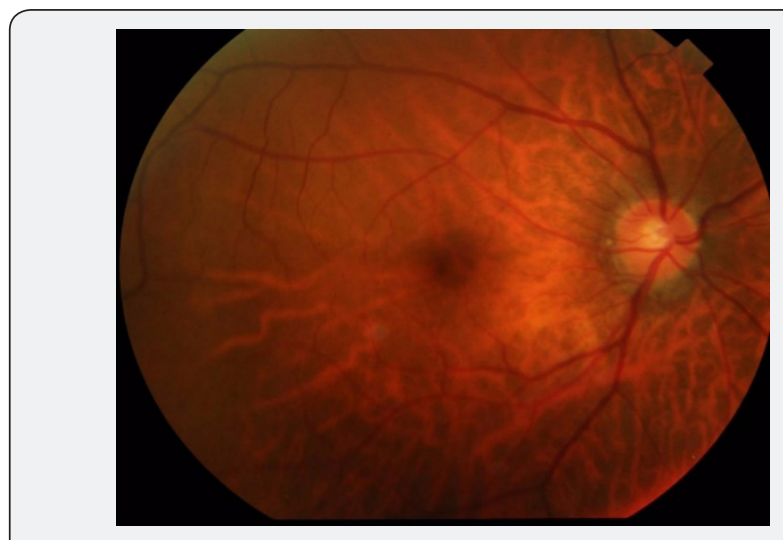

Figure 1: The tessellated fundus with a crescent.

Hypoplasia of the Retinal Pigment Epithelium (RPE) following axial elongation reduces the pigment, allowing the choroidal vessels to be seen. This is referred to as tessellated fundus (Figure 1) [8]. Tessellated fundus is usually associated with a crescent. It is one of the earliest visible signs and is often observed in children [9]. It usually doesn't decrease visual acuity. Highly myopic patients with tessellated fundus alone have less myopia, shorter 


\section{JOJ Ophthalmology}

axial length, and less staphyloma than highly myopic patients with diffuse chorioretinal atrophy [10].

\section{Chorioretinal atrophy}

Tokoro classified chorioretinal atrophy into diffuse and patchy chorioretinal atrophy [8]. Diffuse chorioretinal atrophy appears as an ill-defined yellowish lesion in the posterior fundus of highly myopic patients (Figure 2). It is observed mainly in the macula and around the optic disc. It increases with aging as well as with increased axial length and begins to appear at around the age of 40 . The choroid is severely thinned and is almost absent while the RPE is preserved. Fluorescein angiography shows slight hyper fluorescence in the late phase. Diffuse atrophy tends to progress with the development of the posterior staphyloma. The progression of diffuse atrophy may be the result of continuous mechanical expansion within the staphyloma found in highly myopic eyes. It has a relatively good visual prognosis [8].

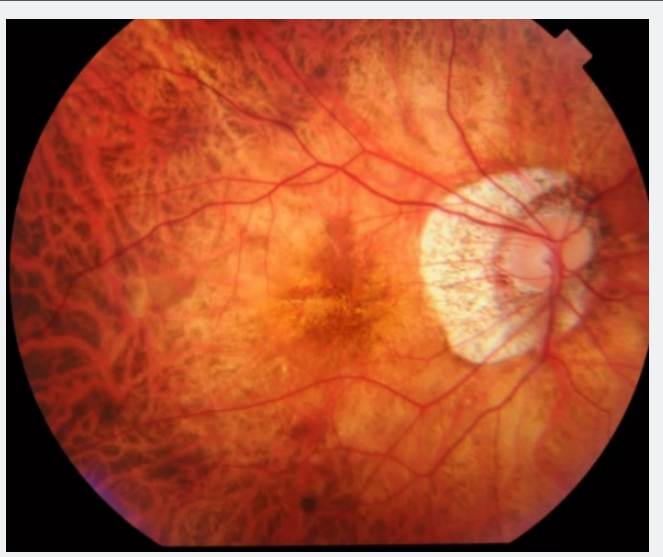

Figure 2: Diffuse chorioretinal atrophy appearing as an illdefined yellowish lesion in the posterior fundus of a high myope with peripapillary atrophy.

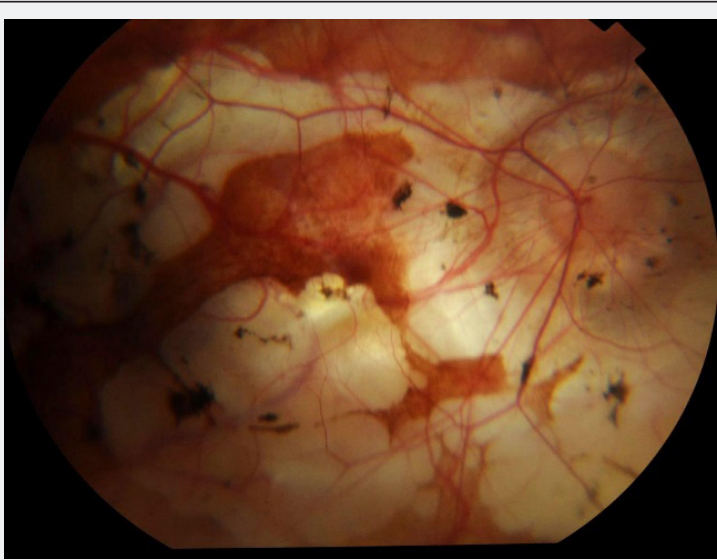

Figure 3: Patchy chorioretinal atrophy consists of grayish white, well defined lesions.

Patchy chorioretinal atrophy is a grayish white, well defined lesion. It is characterized by complete atrophy, in a restricted area, of RPE and choriocapillaris, so that the sclera can be observed through the transparent retina (Figure 3) [8]. Fluorescein angiography shows a choroidal filling defect. With increasing age, areas of patchy atrophy enlarge and coalesce with each other [11]. It is classified into 3 types, with one developing from lacquer cracks, one within the area of diffuse chorioretinal atrophy, and one along the border of a posterior staphyloma [9]. Macular retinoschisis tends to occur preferentially in an area of patchy atrophy [12]. Vision is not impaired unless the macula is involved with the enlargement of patchy atrophy [8].

\section{Lacquer cracks}

Lacquer cracks were first described by Salzmann in 1902 . They are ruptures in the RPE-Bruch's membrane-choriocapillaris complex [13]. Clinically, they appear as fine, irregular, yellow lines in the posterior fundus of high myopes which might be crisscrossing (Figure 4). They occur after subretinal hemorrhage [14]. They are potentially sight threatening as they precede the development of Fuch's spot, Choroidal Neovascularization (CNV), and patchy chorioretinal degeneration. In one study, they were found to be present in $4.3 \%$ of highly myopic eyes [15]. They develop at a relatively early age in highly myopic eyes and show a consistent linear hyper fluorescence during fluorescein angiography (Figure 5). Once lacquer cracks develop in the eye, it tends to develop one after the other in the same eye [6]. In a follow up study of 75 eyes with lacquer cracks, over 5 years 32 eyes (42.7\%) showed an increase in the width of the cracks and these eyes progressed to patchy chorioretinal atrophy, 10 eyes (13.3 \%) developed CNV, and 10 eyes (13.3\%) had an increase in number of lacquer cracks [9].

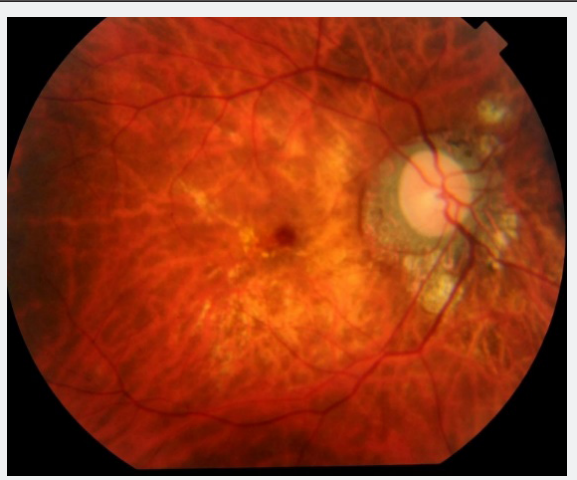

Figure 4: Lacquer cracks are fine irregular yellow lines that crisscross.

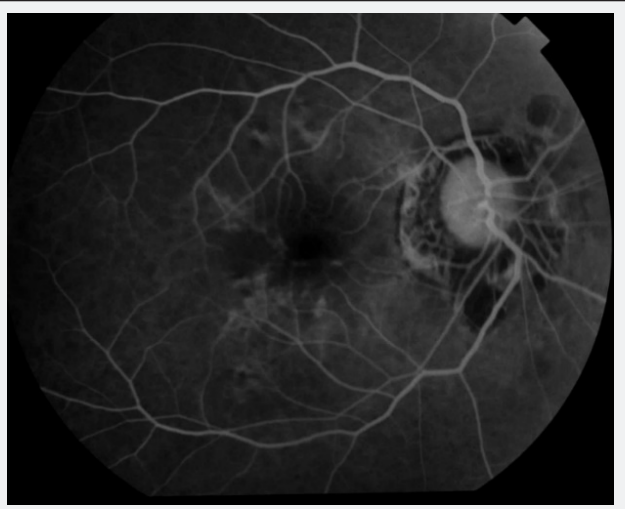

Figure 5: Fluorescein angiography showing linear hyper fluorescence due to lacquer cracks. 


\section{JOJ Ophthalmology}

\section{Fuchs' spot}

Fuchs' spots (Forester-Fuchs spot) are pigmented scars left by choroidal neovascularization. This relation was recognized in 1977 by fluorescein angiography [16]. It was first described by Forester in 1862 and studied by Fuchs in 1901. They appear as central and circular, dark spots, which occur due to proliferation of RPE cells around previous CNV (Figure 6) [6]. It is most frequently observed in middle age and has a female predilection $[11,15]$. Occasionally it is surrounded by an area of chorioretinal atrophy. Its presence denotes a guarded prognosis for visual acuity. It can fade with time [8]. In a Japanese study of 1584 eyes with high myopia, 36.8\% had tessellated fundus, $46.1 \%$ had diffuse atrophy, $8.6 \%$ had patchy atrophy and $8.5 \%$ had Fuchs' spot (Figure 11) [17].

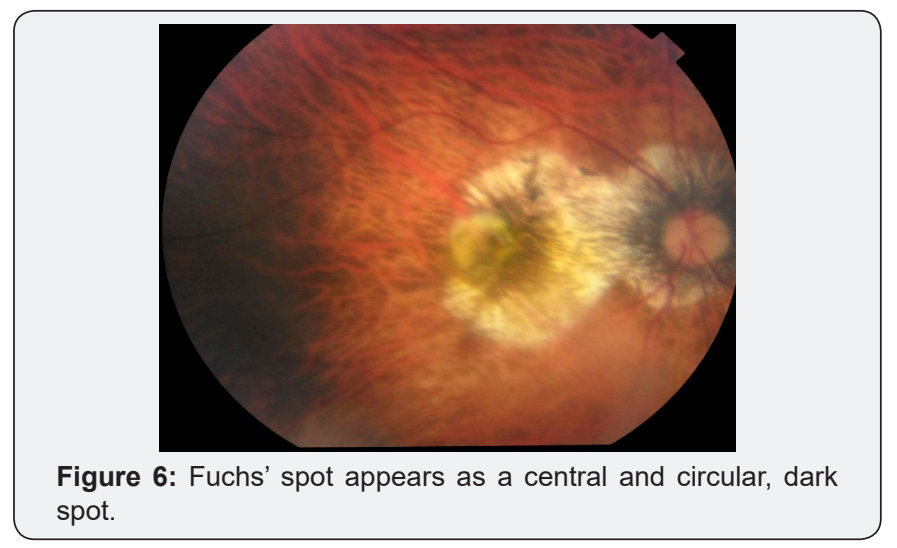

In another Japanese study of 806 highly myopic eyes, 41\% showed progression of myopic retinopathy over a period of 12.7 years. In this study, $13.4 \%$ of patients with tessellated fundus, $49.2 \%$ of those with diffuse atrophy, $69.3 \%$ of those with lacquer cracks, $70.3 \%$ of those with patchy atrophy and $90.1 \%$ of those with CNV, showed progression of their myopic retinopathy. These results suggest that the incidence of progression of myopic retinopathy depends on the type of lesion present [9]. In a Taiwanese eye study, $72.7 \%$ of highly myopic patients had signs of myopic retinopathy. In this study, myopic retinopathy was defined as the presence of lacquer cracks, focal area of deep choroidal atrophy and macular choroidal neovascularization, or geographic atrophy in the presence of high myopia. The definition of myopic retinopathy appears to differ between studies [18].

\section{Posterior staphyloma}

A staphyloma is an outpouching of the wall of the eye that has a radius of less than the surrounding curvature of the wall of the eye (Figure 7). A simple staphyloma is a region that has only 1 radius of curvature. A compound staphyloma is present when there are 2 or more separate staphylomas. Curtin identified 10 types using the indirect ophthalmoscope in a series of 250 patients [19]. Types I-V are simple, while types VI-X are compound. In a study evaluating posterior staphylomas by B-scan ultrasonography, $52.7 \%$ were Type II, $23.4 \%$ were Type I and $17 \%$ were Type IX [20].

In Type V staphyloma, the nerve is usually at the border of the staphyloma and has a tilted appearance. This has led to two different names for this structural configuration: inferior staphyloma or tilted optic disc syndrome [21]. They commonly have visual field defects and choroidal neovascularization with many foci [22]. Not all eyes with progressive myopia will develop a posterior staphyloma, but with increasing axial length, staphyloma development appears to increase. In one study, the prevalence increased from $1.4 \%$ in eyes $26.5-27.4 \mathrm{~mm}$ to $71.4 \%$ in eyes 33.5-36.6mm long [15].

Staphyloma formation in pathological myopia is part of a spectrum of myopic maculopathy, and a variety of features such as retinoschisis, retinal holes and detachments, CNV, and atrophy have been noted to occur in the presence of staphyloma [22]. In one study, the prevalence of macular abnormalities in high myopia, such as foveoschisis, vascular traction, and epiretinal membrane formation, was significantly more frequent in the presence of a posterior staphyloma and $53.65 \%$ of eyes with posterior staphyloma were observed to have macular abnormalities [23]. At the depth of a staphyloma, the retina is stretched over a larger area increasing the stress on the retina. This results in a force acting perpendicularly inward from the retina. Any local traction on the retina through epiretinal membrane or remnants of attached vitreous would increase this force. This can lead to retinoschisis (Figure 7) or retinal detachment [24].

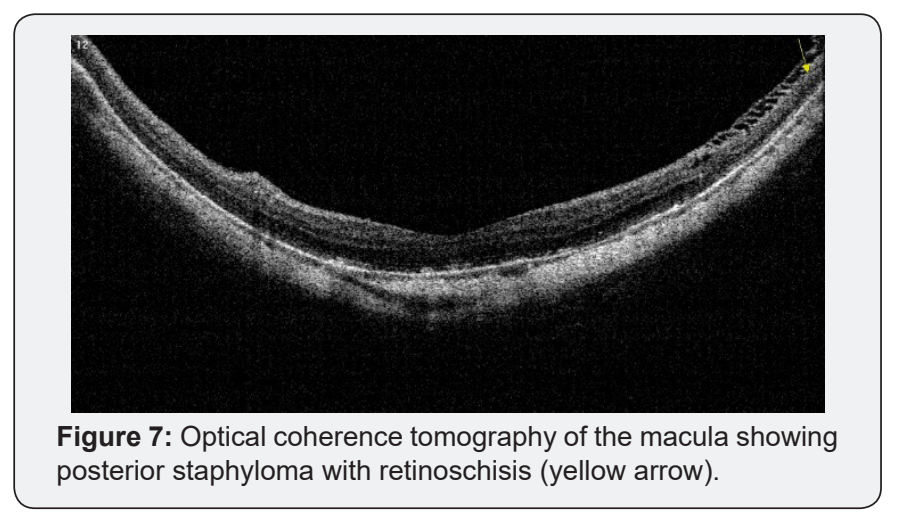

\section{Myopic CNV}

Myopic choroidal neovascularization is the growth of new blood vessels from the choroid to the retina (Figure 8). It is the most common vision-threatening complication among all the myopic fundus pathologies, [25] reported to occur in 5\%-10\% of patients with high myopia [26]. The CNV occurring in high myopia is associated with typical pathological findings such as lacquer cracks and patchy atrophy [9]. Lacquer cracks may offer avenues for the ingrowth of vessels or may indicate a general degeneration of Bruch's membrane such that CNV may be more likely to occur. In one study, $20 \%$ of eyes with patchy atrophy and 29.4\% with lacquer cracks developed CNV [27]. It tends to be smaller in dimension and with a smaller extent of leakage when compared to the age-related form of CNV. Another difference is that in the majority of cases, myopic CNV is located between the neurosensory retina and the RPE, making it a Type $2 \mathrm{CNV}$, whereas the vast majority of AMD-related CNV are classified as Type $1 \mathrm{CNV}$, as they are sub-RPE lesions [28]. 


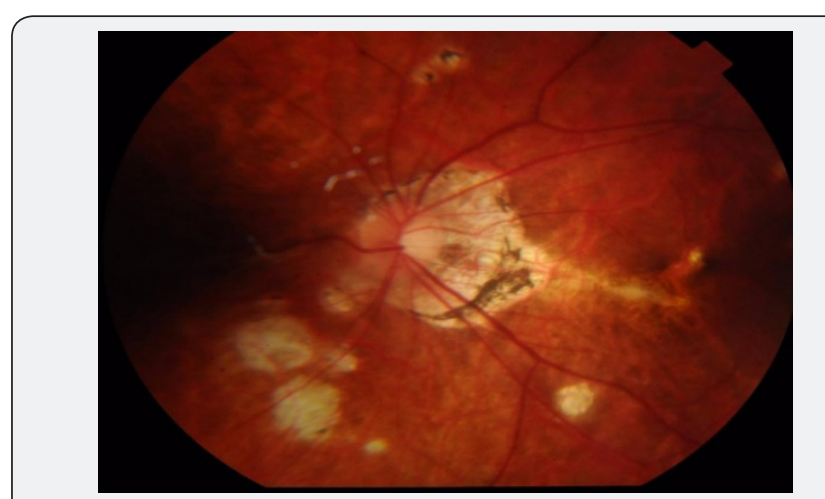

Figure 8: Myopic CNV appearing as a grayish yellow lesion in the macula and associated with a lacquer crack.

In addition to having a low activity, bleeding around myopic CNVs is absorbed quickly, and the neovascular membrane spontaneously shrinks and regresses without the episode of rebleeding in many cases. However, atrophic lesions gradually form around regressed neovascular membranes in highly myopic eyes and lead to further decrease of visual acuity. The development of chorioretinal atrophy around the neovascular membrane is influenced by many factors, including axial length, posterior staphyloma, choroidal circulation in the posterior fundus, or surrounding diffuse atrophy. After the choroidal neovascular membrane completely regresses, it is difficult to differentiate chorioretinal atrophy with regressed neovascular membrane from patchy atrophy without choroidal neovascularization ophthalmoscopically [8].

In a study to clarify the long-term visual outcome of myopic CNV, 27 eyes with myopic CNV were observed without treatment for more than 10 years. At initial examination, $22.2 \%$ of eyes had a visual acuity of greater than $20 / 40$, while $29.6 \%$ of eyes had a visual acuity of less than $20 / 200$. However, at final examination, only one eye retained a visual acuity of greater than $20 / 40$ and $96.3 \%$ of eyes had a visual acuity of less than $20 / 200$ during the follow-up of more than 10 years. At 5 and 10 years after onset, all CNVs were observed to have regressed completely, becoming flat and sometimes unrecognizable. This suggests low activity and a self-limiting course for myopic CNVs. However, chorioretinal atrophy gradually developed around the regressed CNV in 20 of 27 eyes $(74.1 \%)$ by 3 years after initial examination. At 5 years after onset and at final examination, chorioretinal atrophy had developed in 26 of the same 27 eyes (96.3\%). Furthermore, the size of the area of chorioretinal atrophy was noted to gradually enlarge [29].

Fluorescein angiography demonstrates the vascular ingrowth as early hyper fluorescence with variable amounts of leakage later in the angiography. Some eyes show nearly no leakage of fluorescein. Thus, the neovascularization seen in highly myopic eyes can show some but not necessarily all the features required to classify the neovascularization as being classic. The proportion of cases classified as predominantly classic were approximately $80 \%$ in the Verteporfin in Photodynamic Therapy Study Group [30]. Optical Coherence Tomography (OCT) can show signs of exudation, such as intra- or subretinal fluid, being present in eyes with myopic CNV as well as the CNV membrane itself. Small hemorrhages are usually not easy to visualize with OCT. Subretinal scarring may also be seen (Figure 9) [6].

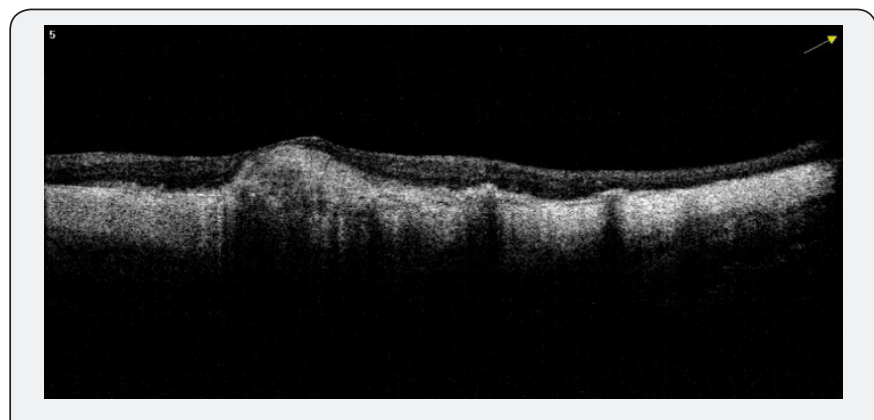

Figure 9: OCT showing a scarred myopic CNV.

The incidence of CNV in high myopia is probably related to a number of factors. These factors include age, refractive status, axial length, gender, and potentially involvement of the fellow eye [15]. Advances in the treatment of CNV secondary to pathologic myopia have trended those from wet AMD in both the proposed theoretical basis and practice, however, the number of studies done on myopic CNV is much less than those on wet AMD [6]. Treatment modalities include laser photocoagulation, transpupillary thermotherapy, photodynamic therapy, intravitreal injections, surgical removal of the choroidal neovascular membrane and macular translocation [31].

\section{Peripheral retinal lesions}

The major peripheral chorioretinal changes associated with pathologic myopia are lattice degeneration, snail track degeneration, white-without pressure, pigmentary degeneration, paving stone degeneration, retinal holes and retinal tears. Each of these entities has a distinct morphology and prevalence varying with age and axial length [32]. Lattice and snail track degenerations are frequently associated with retinal breaks and rhegmatogenous retinal detachments while white-withoutpressure, paving stone, and pigmentary degeneration are usually benign [6]. The dynamic interaction between the vitreous and the retina plays an important role in the development, appearance, and progression of these peripheral retinal changes [6].

\section{a. Lattice degeneration}

Lattice degeneration is a peripheral vitreoretinal thinning that is clinically important because of the potential risk of developing retinal breaks and detachments, with the risk of detachment estimated at about 1 in 90 in lattice degeneration with round holes [33]. In one study, the prevalence of lattice degeneration was $4.5 \%$ in normal eyes and $19 \%$ in myopic eyes with an increase in prevalence in those over -8.00 diopter [34]. In another study of 218 highly myopic patients (436 eyes), the prevalence of lattice degeneration was $33 \%$ and there was an inverse relationship between axial length and the prevalence of lattice degeneration in these myopic eyes [35]. Lattice lesions can vary in their appearance. They can be linear or oval lesions of retinal 


\section{JOJ Ophthalmology}

thinning that can be of various sizes and extents of pigmentation. They are usually at or anterior to the equator and circumferential in distribution (Figure 10). Some may have round atrophic holes within them. They may have branching white lines corresponding to thickened vessels. These white lines are uncommon in young patients but increase with age [36]. By electron microscopy, there is thinning of the retina, fibrosis of blood vessels, loss of retinal neurons, accumulation of extracellular (glial) material, pigment abnormalities, and alterations of the internal limiting membrane [36]. As the margin of lattice degeneration is associated with vitreous adhesions, posterior vitreous detachments can result in a retinal tear along the edges of thelesion (Figure 11). Despite the risk of developing retinal tears and retinal detachments, prophylactic laser of lattice degeneration to prevent retinal detachment is not universally accepted. This is usually recommended if there is a symptomatic retinal tear or a history of retinal detachment in the fellow eye [31]. The etiology is still uncertain, but it is likely that environmental and genetic factors play a role [6].

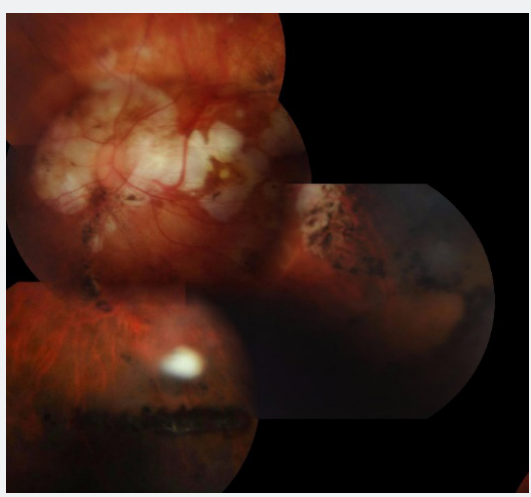

Figure 10: Lattice degeneration with pigmentation, located at the level of the equator and oriented circumferentially parallel to the ora serrata in a highly myopic patient.

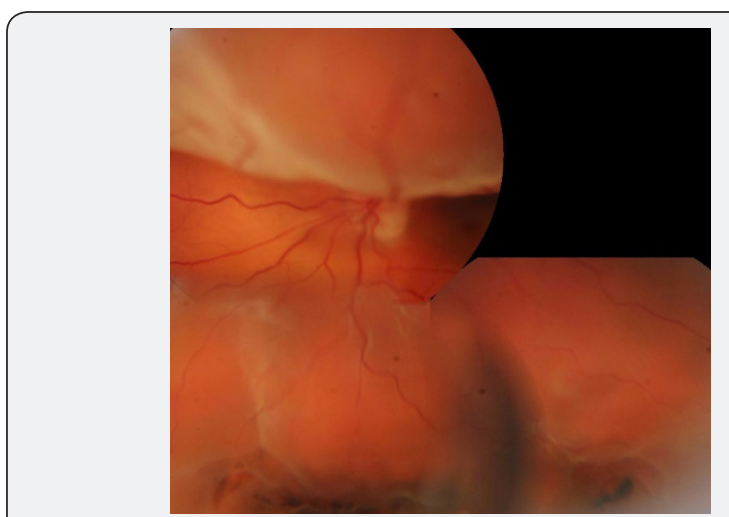

Figure 11: Two horse-shoe retinal tears along the posterior border of a lattice degeneration in a highly myopic patient that resulted in retinal detachment.

\section{b. Snail track degeneration}

Snail-track degenerations are linear nonpigmented lesions that have a glistening, frostlike appearance. There is a controversy as to whether they represent a variant of lattice degeneration or a separate entity [36]. They lack both the white lines and pigmentation and are not commonly seen with lattice degeneration,
[37] but they show the characteristic shape and are located very anteriorly. Like lattice degeneration, they can be associated with round atrophic holes and horseshoe tears [6].

\section{c. White-with pressure}

White-with pressure is a whitish translucent peripheral retinal lesion found between the equator and the ora serrata. It appears with scleral indentation and causes obscuration of the underlying choroid. It does not appear to be associated with retinal breaks. It should be differentiated from a shallow retinal detachment [38].

\section{d. White-without pressure}

White-without pressure refers to a spontaneous opacification of the retina that partially obscures the normal choroidal vascular color and pattern (Figure 12). It tends to run circumferentially, behind the ora, but may reach the equator. It can be flat or have a slightly elevated appearance. It often covers diffusely almost the entire retinal periphery but can also appear as smaller focal patches. It is more prevalent in young patients [39]. In a Chinese study of 337 highly myopic patients, its prevalence was found to be $21.1 \%$ [33] It is postulated to be due to areas of vitreoretinal traction. It is essentially a benign lesion [6].

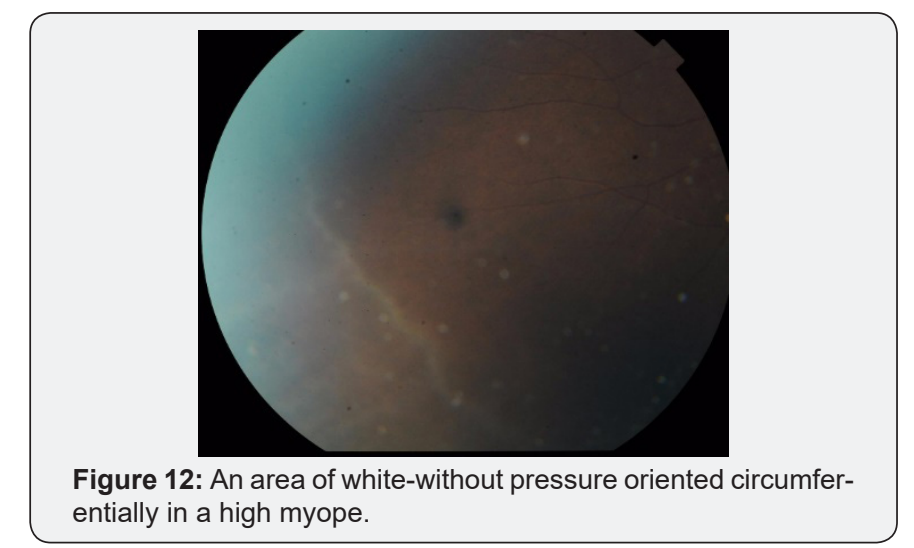

e. Pigmentary degeneration

Pigmentary degeneration corresponds to a variable degree of pigmentation in the extreme periphery. Its prevalence increases with both age and axial length. In a Chinese study, it was found to be the most common peripheral retinal lesion in high myopes, with a prevalence of $37.7 \%$ in 337 patients [32]. In a study of fellow eyes of patients with retinal detachment, $32 \%$ of patients with pigmentary degeneration had retinal breaks [40]. It appears to be an essentially benign lesion however and its morbidity may be attributed to unidentified underlying areas of lattice degeneration prone to formation of retinal breaks [36].

\section{f. Paving stone degeneration}

The term paving stone degeneration can be used interchangeably with cobblestone degeneration. They usually appear as small, sharply demarcated, flat or slightly depressed, rounded, yellow to whitish areas of depigmentation and retinal thinning, with subsequent increased visualization of the relatively preserved underlying major choroidal vessels [38]. They are usually located one or two-disc diameters posterior to the ora 


\section{JOJ Ophthalmology}

serrata. They can occur singly or in groups. When in group, they have a tendency to coalesce. They were found with a rate of $27.1 \%$ in a study of 513 eyes with axial length greater than $24 \mathrm{~mm}$ [39]. It is not significantly associated with retinal breaks [6].

\section{g. Retinal breaks}

A retinal break is a full thickness defect in retinal continuity which can be either a hole or a tear. Retinal holes are related to chorioretinal degenerative changes while retinal tears result from the traction of adherent vitreous on a weakened retinal area. In a study of 337 highly myopic Chinese adults, 6.2\% had retinal breaks [32]. Retinal tears can be flap (horseshoe) (Figure 11) or operculated tears. They can be symptomatic or asymptomatic. Symptoms include light flashes and floaters. Tears occur preferentially in the upper and temporal halves [41]. Anatomically, they can be classified as oral (dialysis), intra, extra and juxtabasal, which carry the highest risk of retinal detachment. Juxtabasal tears are typically flap tears which occur following acute posterior vitreous detachment with traction exerted from the posterior border of the vitreous base on the anterior margins of the flap tear [38].

Atrophic retinal holes are small and usually located with lattice lesions. They usually occur early in life and without producing symptoms, as they are caused by a localized dissolution of tissue in the very thin base of the lattice. They are more common than retinal tears but less frequently lead to retinal detachment [36]. In one study, $2.8 \%$ of all primary retinal detachments were due to round holes of lattice lesions. These detachments were more common in younger and myopic patients [42]. Symptomatic flap tears are the only lesions with a strong evidence-based recommendation for systematic prophylactic therapy [43]. Treatment is with three rows of laser if feasible or cryotherapy. Asymptomatic flap tears in myopic, aphakic and fellow eyes of patients who had a retinal detachment are sometimes treated [43].

\section{Rhegmatogenous retinal detachment}

The risk of retinal detachment in myopic eyes is 3 to 8 times that in hyperopic and emmetropic eyes [44]. In one study, myopia was present in $67 \%$ of eyes with detachments [31]. According to The Eye Disease Case Control Study Group, [45] the relationship between risk of detachment and amount of myopia is linear, with 1-3 D of myopia being associated with four times increased risk, and greater than $3 \mathrm{D}$ having a tenfold increased risk. The relative risk was higher in younger patients. The occurrence of Rhegmatogenous Retinal Detachments (RRD) requires the presence of retinal breaks, some degree of vitreousliquefaction and persistent vitreoretinal traction in the region of retinal breaks. The usual sequence of vitreous changes that lead to RRD begins with vitreous liquefaction leading to increased mobility and decreased stability of the vitreous gel. This is followed by Posterior Vitreous Detachment (PVD). Myopic eyes have a substantially increased liquid component of the vitreous gel compared to emmetropic and hyperopic eyes with subsequent decrease in vitreous stability. This results in an increased frequency of PVD at relatively young ages [46].
In the most common forms of RRD, separation of the cortical vitreous from the retinal surface is not completed at sites of abnormal vitreoretinal adhesions, and flap (horseshoe) tears develop at such locations [38]. Vitreous traction upon lattice lesions containing atrophic holes is another form of common retinal detachment. Any collection of fluid greater than 1-disc diameter is generally considered to be a retinal detachment, although collections of about 1 DD are sometimes called subclinical detachments [6]. Vitreoretinal adhesions can be considered as forms of peripheral vitreoretinal degeneration. Most of these zones are invisible, but many such as lattice degeneration are quite apparent. Relatively strong adhesions appear to be present in normal relatively youthful eyes, and so when PVD occurs at a relatively young age in highly myopic eyes, [47] these adhesions might cause a retinal tear [6].

Retinal detachment resulting from Full-Thickness Macular Holes (FTMH) occurs most commonly in highly myopic eyes (Figure 13) [48]. This might be due to the presence of posterior staphyloma and probably also a weak RPE-photoreceptor adherence [49]. In a study of 52 highly myopic eyes with FTMH, extensive RD was present in 71\% [50]. The incidence of RRD with FTMH increases with increasing myopic refractive error, chorioretinal atrophy and with the presence of a staphyloma [51]. Two patterns for FTMH formation have been described based on OCT. In pattern 1, a focal area of the external retinal layer overlying the RPE is elevated, followed by the development of an Outer Lamellar Macular Hole (OLMH) and RRD. The OLMH and RD then gradually enlarge until the OLMH becomes attached to the overlying retinal layers. A FTMH finally develops when the roof of RD opened. In pattern 2, an inner lamellar macular hole (ILMH) progresses directly to a FTMH [52].

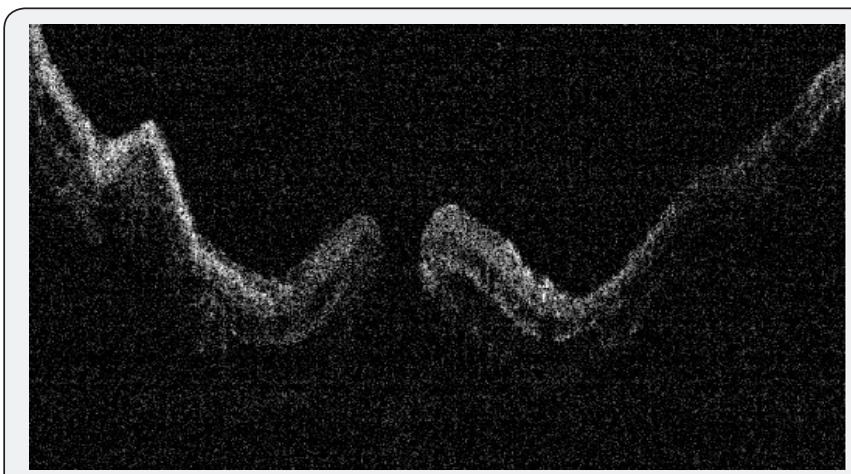

Figure 13: Full-thickness macular hole with retinal detachment by OCT.

Foveal retinal detachment can also occur without the development of MH. In a study of 78 eyes with staphyloma by OCT 9\% had foveal retinal detachment without FTMH [12]. Although controversy regarding the choice of surgical technique for RRD is widespread, surgeons generally agree on the three basic steps for closing retinal breaks and reattaching the retina, which are examination and location of all retinal breaks, creating a controlled injury to the RPE and retina to form chorioretinal adhesions surrounding all breaks, and employing a technique to approximate the retinal breaks to the underlying treated RPE. 


\section{JOJ Ophthalmology}

This can be achieved by either scleral buckling, vitrectomy, or in some cases, pneumatic retinopexy [6]. Traditional scleral buckling has served as a successful technique since the 1950s. However, more recent developments have produced more comprehensive methods for retinal reattachment surgery from which the surgeon may select the appropriate procedure for each case [6].

\section{Myopic Macular Retinoschisis (MRS)}

As a consequence of the ectasia secondary to the posterior staphyloma, highly myopic individuals can develop retinoschisis (Figure 14). This is the splitting of the retinal layers in the macula, most commonly in the outer plexiform layer (outer retinoschisis) [53]. Myopic retinoschisis was recently described by OCT. Until then, this type of retinoschisis had been poorly characterized and was probably misinterpreted clinically [54]. OCT features are varied but typically include columnar bridging structures within the schisis cavity, and a variable degree of vitreo-retinal traction including internal limiting membrane dehiscence (Figure 14) [54]. Most of the patients with MRS may be relatively asymptomatic especially while the eyes do not develop more serious complications like full-thickness $\mathrm{MH}$, and MRS may persist for many years before affecting vision significantly [12].

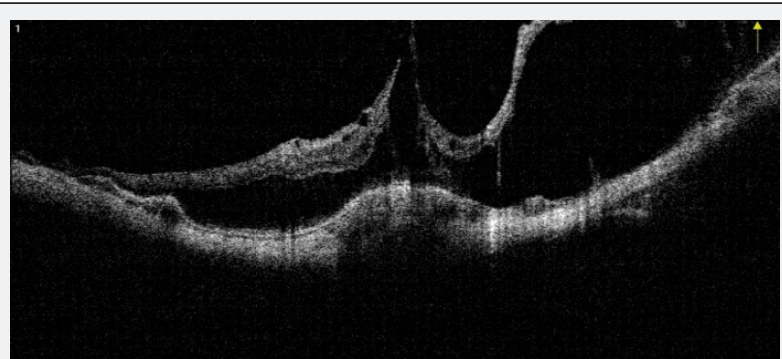

Figure 14: OCT showing macular retinoschisis with inner lamellar hole in the central fovea.

Various macular lesions coexist in eyes with MRS, such as lamellar macular hole (lamellar MH), Full-Thickness Macular Hole (FTMH), and foveal Retinal Detachment (RD). In a study of 21 eyes with MRS, 28.6\% developed foveal RD, and 9.5\% developed FTMH during follow up [53]. Although the presence of MRS can be suspected ophthalmoscopically in some cases, OCT is necessary for diagnosis [6]. It is caused by various factors including vitreomacular traction (from perifoveal PVD), remnant cortical vitreous layer (after PVD), epiretinal membrane, and intrinsic noncompliance of the internal limiting membrane (ILM) [55]. In a study using electron microscopy, collagen fiber and cell debris were identified on the inner surface of ILM in $70 \%$ of the eyes with MRS, whereas none in ILM from control subjects (idiopathic $\mathrm{MH}$ ) [56]. Retinal arteriolar stiffness was also suggested as a minor mechanism contributing to the MRS development [55]. Recent studies using swept-source OCT support an association between scleral curvature alterations and MRS [57]. The usefulness of pars plana vitrectomy in resolving the foveal retinal detachment and MRS has been reported in several studies. The majority of eyes with MRS without foveal RD retain relatively good vision, so timing of surgery for MRS without foveal RD is uncertain [58].

\section{Conclusion}

Various posterior segment manifestations may develop in patients with pathological myopia. Distinguishing these manifestations clinically and by multimodal imaging is essential to determine the management and prognosis of these conditions.

\section{References}

1. Elnahry AG, Khafagy MM, Esmat SM, Mortada HA (2018) Prevalence of pathological myopia among patients in a large tertiary care center in Egypt. New Front Ophthalmol, DOI : 10.15761/NFO.1000216.

2. Gawdat I (1976) Studies on the incidence of refractive errors in Egypt. Bull Ophthalmol Soc Egypt 69(73): 513-520.

3. Hammond CJ, Snieder H, Gilbert CE, Spector TD (2001) Genes and environment in refractive error: the twin eye study. Invest Ophthalmol Vis Sci 42(6): 1232-1236.

4. Duke Elder S (1970) Pathological Refractive Errors. In: Duke Elder S (Ed.), St Louis, Mosby, USA, pp. 297-373.

5. Tokoro T (1988) On the definition of pathologic myopia in group studies. Acta Ophthalmol 185: 107-108.

6. Spaide RF, Ohno Matsui K, Yannuzzi LA (2014) Pathologic Myopia. In: Spaide RF, Ohno Matsui K, Yannuzzi LA (Eds.), Springer, New York, USA, pp. 105-109.

7. Vongphanit J, Mitchell P, Wang JJ (2002) Prevalence and progression of myopic retinopathy in an older population. Ophthalmology 109(4): 704-711.

8. Tokoro $\mathrm{T}$ (1998) Atlas of posterior fundus changes in pathologic myopia. Springer, New York, USA, pp. 5-22.

9. Hayashi K, Ohno Matsui K, Shimada N, Moriyama M, Kojima A, et al. (2010) Long-term pattern of progression of myopic maculopathy: a natural history study. Ophthalmology 117: 1595-1611.

10. Wang NK, Lai CC, Chu HY, Chen YP, Chen KJ, et al. (2012) Classification of early dry-type myopic maculopathy with macular choroidal thickness. Am J Ophthalmol 153(4): 669-677.

11. Curtin B (1985) Basic science and clinical management. In: Curtin B (Ed.), The myopias. Harper \& Row, Philadelphia, pp. 237-245.

12. Baba T, Ohno Matsui K, Futagami S, Yoshida T, Yasuzumi K, et al. (2003) Prevalence and characteristics of foveal retinal detachment without macular hole in high myopia. Am J Ophthalmol 135(3): 338-342.

13. Salzmann M (1902) The choroidal changes in high myopia. Arch Ophthalmol 31: 41-42.

14. Klein RM, Green S (1988) The development of lacquer cracks in pathologic myopia. Am J Ophthalmol 106(3): 282-285.

15. Curtin BJ, Karlin DB (1970) Axial length measurements and fundus changes of the myopic eye. I. The posterior fundus. Trans Am Ophthalmol Soc 68: 312-334.

16. Levy JH, Pollock HM, Curtin BJ (1977) The Fuch's spot: an ophthalmoscopic and fluorescein angiographic study. Ann Ophthalmol. 9(11): 1433-1443.

17. Tokoro T (1994) Mechanism of axial elongation and chorioretinal atrophy in high myopia. Nippon Gannka Gakkai Zasshi 98(12): 12131237.

18. Chen SJ, Cheng CY, Li AF, Peng KL, Chou P, et al. (2012) Prevalence and associated risk factors of myopic maculopathy in elderly Chinese: the Shihpai Eye Study. Invest Ophthalmol Vis Sci 53(8): 4868-4873.

19. Curtin B (1977) The posterior staphyloma of pathologic myopia. Trans Am Ophthalmol Soc 75: 67-86.

20. Hsiang HW, Ohno Matsui K, Shimada N, Hayashi K, Moriyama M, et al. (2008) Clinical characteristics of posterior staphyloma in eyes with 


\section{JOJ Ophthalmology}

pathologic myopia. Am J Ophthalmol 146(1): 102-110.

21. Young SE, Walsh FB, Knox DL (1976) The tilted disk syndrome. Am J Ophthalmol 82(1): 16-23.

22. Quaranta M, Brindeau C, Coscas G, Soubrane G (2000) Multiple choroidal neovascularizations at the border of a myopic posterior macular staphyloma. Graefes Arch Clin Exp Ophthalmol 238(1): 101103.

23. Henaine Berra A, Zand Hadas IM, Fromow Guerra J, Garcia Aguirre G (2013) Prevalence of macular anatomic abnormalities in high myopia. Ophthalmic Surg Lasers Imaging Retina 44(2): 140-144.

24. Spaide RF, Fisher Y (2005) Removal of adherent cortical vitreous plaques without removing the internal limiting membrane in the repair of macular detachments in highly myopic eyes. Retina 25(3) 290-295.

25. Avila MP, Weiter JJ, Jalkh AE, Trempe CL, Pruett RC, et al. (1984) Natura history of choroidal neovascularization in degenerative myopia. Ophthalmology 91(12): 1573-1581.

26. Grossniklaus HE, Green WR (1992) Pathologic findings in pathologic myopia. Retina 12(2): 127-133.

27. Ohno Matsui K, Yoshida T, Futagami S, Yasuzumi K, Shimada N, et al. (2003) Patchy atrophy and lacquer cracks predispose to the development of choroidal neovascularisation in pathological myopia Br J Ophthalmol 87(5): 570-573.

28. Grossniklaus HE, Gass JD (1998) Clinicopathologic correlations of surgically excised type 1 and type 2 submacular choroidal neovascular membranes. Am J Ophthalmol 126(1): 59-69.

29. Yoshida T, Ohno Matsui K, Ohtake Y, Takashima T, Futagami S, et al. (2002) Long-term visual prognosis of choroidal neovascularization in high myopia. Ophthalmology 109(4): 712-719.

30. Verteporfin in Photodynamic Therapy Study Group. Photodynamic therapy of subfoveal choroidal neovascularization in pathologic myopia with verteporfin 1-year results of a randomized clinical trial VIP report no. 1. Ophthalmology 108: 841-852.

31. Beuerman RW, Saw SM, Tan DTH, Wong TY (2010) Myopia: Animal models to clinical trials. World Scientific, Singapore, pp. 3-21.

32. Lai TY, Fan DS, Lai WW, Lam DS (2008) Peripheral and posterior pole retinal lesions in association with high myopia: a cross-sectional community-based study in Hong Kong. Eye (Lond) 22(2): 209-213.

33. Murakami Nagasako F, Ohba N (1983) Phakic retinal detachment associated with atrophic hole of lattice degeneration of the retina Graefe's archive for clinical and experimental ophthalmology. Albrecht von Graefes Archiv fur klinische und experimentelle Ophthalmologie 220(4): 175-178.

34. Cambiaggi A (1968) Research on the role of myopic chorioretinal changes in the pathogenesis of retinal detachment. Ophthalmologica 156(2): 124-132.

35. Celorio JM, Pruett RC (1991) Prevalence of lattice degeneration and its relation to axial length in severe myopia. Am J Ophthalmol 111(1): 20-23.

36. Byer NE (1979) Lattice degeneration of the retina. Surv Ophthalmol 23(4): 213-248

37. Aaberg TM, Stevens TR (1972) Snail track degeneration of the retina. Am J Ophthalmol 73(3): 370-376.

38. Zinn KM (1988) Clinical atlas of peripheral retinal disorders. Springer Verlag, New York, USA.

39. Pierro L, Camesasca FI, Mischi M, Brancato R (1992) Peripheral retinal changes and axial myopia. Retina 12(1): 12-17.

40. Everett WG (1963) The fellow-eye syndrome in retinal detachment.
Am J Ophthalmol 56: 739-748.

41. Tulloh CG (1965) Distribution of holes and tears in primary retinal detachment. Br J Ophthalmol 49(8): 413-431.

42. Tillery WV, Lucier AC (1976) Round atrophic holes in lattice degeneration - an important cause of phakic retinal detachment. Trans Sect Ophthalmol Am Acad Ophthalmol Otolaryngol 81(3 Pt 1): 509518.

43. Wilkinson CP (2000) Evidence-based analysis of prophylactic treatment of asymptomatic retinal breaks and lattice degeneration. Ophthalmology 107(1): 12-15.

44. Austin KL, Palmer JR, Seddon JM, Glynn RJ, Rosenberg L, et al. (1990) Case-control study of idiopathic retinal detachment. Int J Epidemiol 19(4): 1045-1050.

45. (1993) The Eye Disease Case Control Study Group. Risk factors for idiopathic rhegmatogenous retinal detachment. Am J Epidemiol 137: 749-757.

46. Sebag J (1998) Myopia effects upon vitreous-significance in retinal detachments. In: Stirpe M (Ed.), Anterior and posterior segment surgery: mutual problems and common interests. Ophthalmic Communications Society, New York, USA, pp. 366-372.

47. Akiba J (1993) Prevalence of posterior vitreous detachment in high myopia. Ophthalmology 100(9): 1384-1388.

48. Ortis E, Avitabile T, Bonfiglio V (2012) Surgical management of retinal detachment because of macular hole in highly myopic eyes. Retina 32(9): 1704-1718.

49. Negi A, Marmor MF (1986) Quantitative estimation of metabolic transport of subretinal fluid. Invest Ophthalmol Vis Sci 27(11): 15641568.

50. Akiba J, Konno S, Yoshida A (1999) Retinal detachment associated with a macular hole in severely myopic eyes. Am J Ophthalmol 128(5): 654655.

51. Morita H, Ideta H, Ito K, Yonemoto J, Sasaki K, et al. (1991) Causative factors of retinal detachment in macular holes. Retina 11(3): 281-284

52. Sun CB, Liu Z, Xue AQ, Yao K (2010) Natural evolution from macular retinoschisis to full-thickness macular hole in highly myopic eyes. Eye (Lond) 24(12): 1787-1791.

53. Fujimoto M, Hangai M, Suda K, Yoshimura N (2010) Features associated with foveal retinal detachment in myopic macular retinoschisis. Am J Ophthalmol 150(6): 863-870.

54. Takano M, Kishi S (1999) Foveal retinoschisis and retinal detachment in severely myopic eyes with posterior staphyloma. Am J Ophthalmol 128(4): 472-476.

55.Johnson MW (2012) Myopic traction maculopathy: pathogenic mechanisms and surgical treatment. Retina 32(2): S205-S210.

56. Bando H, Ikuno Y, Choi JS, Tano Y, Yamanaka I, et al. (2005) Ultrastructure of internal limiting membrane in myopic foveoschisis. Am J Ophthalmol 139(1): 197-199.

57. Ohno Matsui K, Akiba M, Modegi T, Tomita M, Ishibashi T, et al. (2012) Association between shape of sclera and myopic retinochoroidal lesions in patients with pathologic myopia. Invest Ophthalmol Vis Sci 53(10): 6046-6061.

58. Ikuno Y, Sayanagi K, Ohji M, Kamei M, Gomi F, et al. (2004) Vitrectomy and internal limiting membrane peeling for myopic foveoschisis. Am J Ophthalmol 137(4): 719-724. 
This work is licensed under Creative Commons Attribution 4.0 License DOI: 10.19080/JOJO.2019.07.555709

\section{Your next submission with Juniper Publishers will reach you the below assets}

- Quality Editorial service

- Swift Peer Review

- Reprints availability

- E-prints Service

- Manuscript Podcast for convenient understanding

- Global attainment for your research

- Manuscript accessibility in different formats

( Pdf, E-pub, Full Text, Audio)

- Unceasing customer service

Track the below URL for one-step submission https://juniperpublishers.com/online-submission.php 